\title{
Introduction à la
}

Boîte à outils

d'Avenirs

Immigrants pour les

leaders municipaux

Un regard nouveau sur

le développement économique local 


\section{Le Projet Avenirs Immigrants}

Avenirs Immigrants met au jour de nouvelles perspectives sur les défis et les opportunités que l'immigration peut poser pour les communautés canadiennes, qu'elles soient petites, moyennes ou régionales. Le projet est présenté en partenariat avec le Service du développement économique de la Ville de Hamilton, la Ville de Moncton, le Halifax Partnership, le Leeds-Grenville Local Immigration Partnership et Embauches Immigrants - Magnet, avec l'appui d'Immigration, Réfugiés et Citoyenneté Canada (IRCC).

Avenirs Immigrants est un projet du programme Villes de Migration, qui fait maintenant partie du programme Chaire d'excellence en recherche du Canada sur la migration et l'intégration à l'Université Ryerson. Le programme CERC sur la migration et l'intégration s'avère une source de connaissances utiles et novatrices nous permettant de mieux comprendre des questions contemporaines relatives à la migration et lintégration au Canada et dans le monde entier.

Le programme Villes de Migration apporte une perspective urbaine à sa conception de la bonne pratique dans le domaine d'intégration. Il cherche à promouvoir une échange de connaissances de ville en ville et à tisser des liens entre les nombreux acteurs qui participent au travail pratique quotidien pour assurer que l'intégration soit un composant clé de la réussite urbaine à l'échelle internationale. Consultez notre site Web pour parcourir une série de pratiques prometteuses concernant l'intégration. Là aussi vous trouverez un ensemble intégré d'outils qui peuvent servir à des professionnels au niveau municipal et à des réseaux communautaires ou de bailleurs de fonds se spécialisant dans la migration et l'intégration urbaine à forger des liens plus solides et rendre plus efficaces les pratiques locales d'intégration.

\section{Remerciements}

Ce document a été rédigé par Jenna Blower, sous la direction de Kim Turner, anciennement responsable du programme Villes de Migration, et finalisé sous la supervision d'Anna Triandafyllidou, Chaire d'excellence en recherche du Canada sur la migration et l'intégration.

\section{Pour plus de renseignements :}

cerc.migration@ryerson.cal CERC Migration, Université Ryerson

Canada Excellence Research Chair in Migration \& Integration
Cities of Migration

\section{HALIFAX PARTNERSHIP

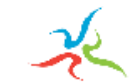

$M O N C T O N$
Immigration, Refugees

Immigration, Réfugiés and Citizenship Canada et Citoyenneté Canada 


\section{Historique}

Au cours de l'année dernière, le projet Avenirs Immigrants a fait des recherches, mené des consultations communautaires en Ontario et dans la région de l'Atlantique, recueilli des pratiques prometteuses et fait usage des forums de webinaires pour apprendre sur le terrain et décortiquer quelques-uns des défis présentés par l'attirance et la rétention d'immigrants dans des petites villes canadiennes.

Le projet Avenirs Immigrants est un cadre de planification communautaire multipartite qui, avec des outils associés, a été développé pour aider des villes petites et moyennes à élaborer des stratégies plus efficaces d'attirance et de rétention d'immigrants.

Nous adoptons une approche pancommunautaire en ce qui concerne la conception d'une économie inclusive. Une telle approche a besoin d'un leadership fort et d'un engagement multipartite dans un vaste champ d'action. Il faut que l'ensemble de la communauté s'implique dans un processus de planification qui est ouvert et inclusif et qui met en valeur la santé et le bienêtre à long terme de tous les résidents.

Le projet Avenirs Immigrants considère l'immigration comme une stratégie critique de développement économique face au déclin de la santé et le bien-être de petites villes au Canada.

L'argument économique en faveur de l'immigration est d'une stabilité à toute épreuve.

La stratégie adoptée, à des fins économiques, pour attirer des immigrants au Canada s'est inspirée de données bien établies démontrant la contribution positive faite par l'immigration à la croissance de l'économie. On se trouve dans un dilemme cependant par rapport à la dispersion homogène d'immigrants partout au pays. Dans les communautés régionales comprenant plusieurs villes petites et moyennes de diverses provinces la population immigrante continue d'être d'une densité relativement faible ; souvent les immigrants y représentent moins de $10 \%$ de la population résidente. Ces communautés enregistrent un faible taux de natalité, c'est à dire elles connaissent une croissance démographique stagnante, ainsi que des industries en évolution rapides et des taux d'émigration élevés. Il est évident que le nombre de jeunes sortant du système local d'éducation ne suffit pas pour répondre aux besoins du marché du travail, sans parler de la croissance économique future. Une solution à cette urgence économique serait d'augmenter le nombre d'immigrants qui s'installent en-dehors des grandes villes. De cette manière, d'un seul coup on pourrait s'attaquer au déclin de la population, favoriser le développement régional, atteindre une distribution plus équitable du capital social et économique et réduire la pression exercée sur les villes canadiennes les plus grandes où la majorité d'immigrants choisissent de s'installer.

Notre expérience de l'initiative Villes de Migration nous a enseigné que " toute ville a la capacité d'être accueillante et de contribuer au succès d'immigrants en créant les conditions d'investissement, d'attachement et d'appartenance " (Villes de Migration 2012, 45).

Cela fait bien plus de deux décennies que des stratégies de régionalisation ont été mises en vigueur avec une gamme de soutiens d'immigration disponibles aux niveaux fédéral et provinciaux. Toutefois, la plupart des programmes et des services se situent dans des villes bien accoutumées à l'installation d'immigrants et fortement axée sur leur intégration. Dans des villes plus petites et moyennes il y a eu depuis plusieurs générations une croissance naturelle relativement homogène de la population. Aujourd'hui, face au besoin urgent de relancer cette croissance grâce à l'immigration, ces villes sans antécédents de l'accueil et l'intégration d'immigrants se trouvent sous une pression accrue de dresser le plan de leur propre avenir immigrant.

La question se pose : comment assurer que ça marche pour les villes canadiennes plus petites? Qu'est-ce qui contribue au succès d'une installation et d'une intégration ? Ou à l'échec par rapport à l'attirance et la rétention? 


\section{Le Cadre d'Avenirs Immigrants}

En vue d'identifier les éléments d'une approche pratique et réussie, nous avons examiné des différents modèles communautaires en provenance du Canada, des États-Unis et de l'Allemagne où l'on s'est efforcé d'intégrer le développement économique et les stratégies d'immigration.

Notre examen nous a induit à proposer un modèle à quatre étapes :

1) exploiter le leadership municipal ;

2) accroître la sensibilisation et la participation de toute la communauté ;

3) mobiliser des intervenants divers ;

4) élaborer des outils pratiques pour aider dans la conception d'une stratégie particulière.

Le résultat de cette approche est spécialement adapté aux besoins et aux actifs de chaque ville.

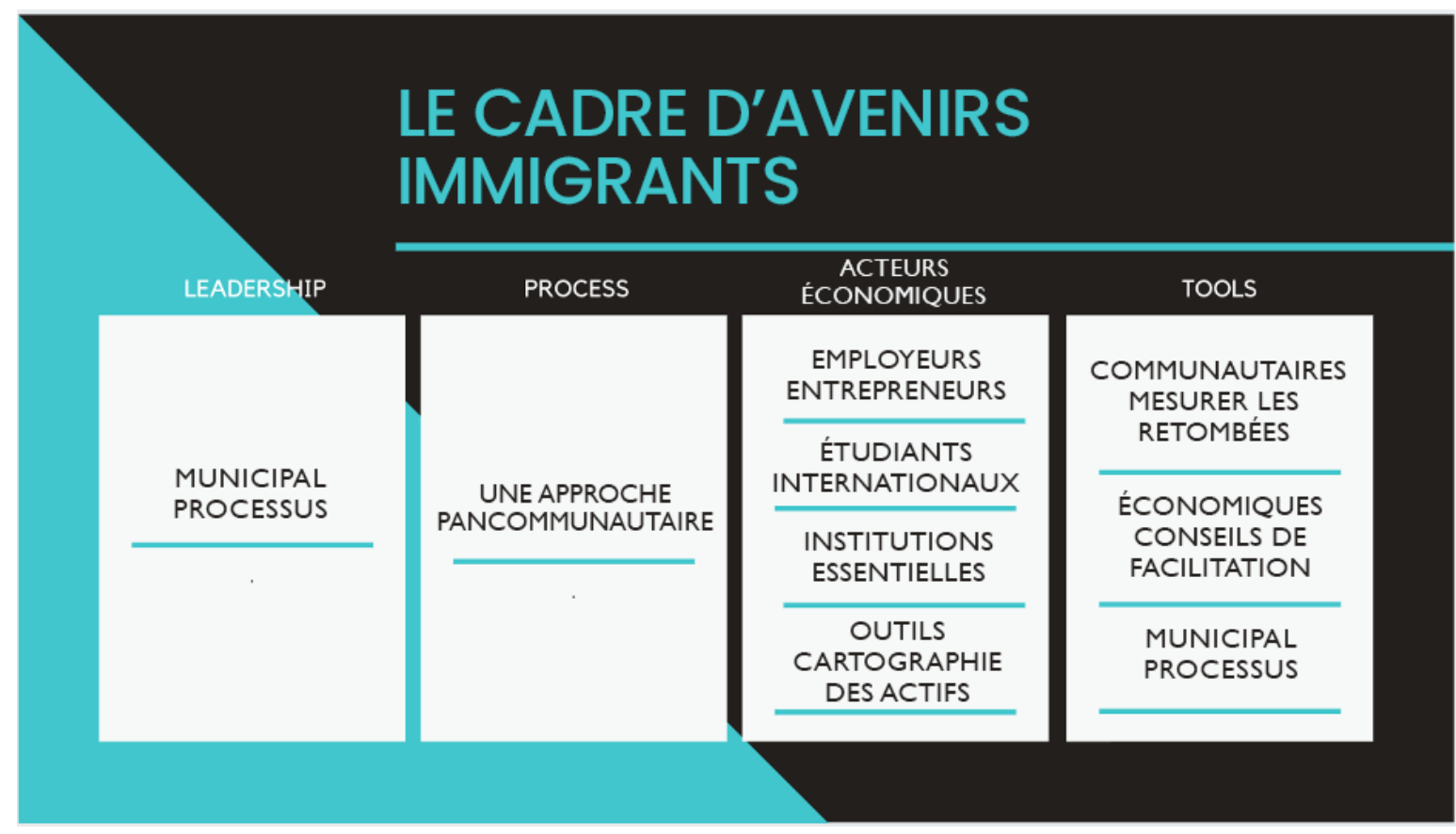

Nous nous sommes rendu compte que les modèles communautaires qui cherchent à aligner le développement économique sur les agendas d'immigration sont plus efficaces quand ils ont reçu l'approbation d'un haut fonctionnaire et quand, en se servant d'une approche pancommunautaire, ils ont assuré la participation de multiples intervenants. Cette approche implique non seulement des acteurs économiques, tels que les employeurs ou les entrepreneurs, mais aussi les institutions d'éducation post-secondaire, les bibliothèques, les prestataires de services de santé, les associations sportives et les étudiants internationaux eux-mêmes, surtout quand il n'y a aucun organisme de soutien consacré aux immigrants. Une telle approche favorise, par surcroît, la création d'une infrastructure de soutien spécialement conçue pour des nouveaux arrivants. II en ressort enfin que le bureau municipal, l'hôpital, la bibliothèque et les principaux employeurs de la ville, par exemple, sont capables de desservir une communauté en pleine croissance et aux besoins divers. Dans la Boîte à outils d'Avenirs Immigrants des autorités locales intéressées vont trouver de quoi les aider à repérer les actifs de leur communauté, en augmenter la sensibilisation, plaider en faveur d'un avenir immigrant et former un partenariat civil pour l'immigration. Nous abordons ci-dessous les éléments variés de l'approche pancommunautaire proposée en matière de la planification de l'immigration. 


\section{Exploiter le leadership municipal}

Notre cadre reconnaît le rôle grandissant de villes dans la consolidation de la capacité politique d'attirer et retenir des immigrants. Elles peuvent aussi devenir des chefs de file en persuadant la communauté d'accueil dans son ensemble à collaborer au soutien de nouveaux arrivants.

Le projet Avenirs Immigrants a été conçu pour permettre aux leaders urbains et communautaires d'instaurer un processus de planification où s'alignent les agendas des politiques d'économie et d'immigration. On est conscient qu'un grand nombre d'intervenants municipaux et communautaires y jouent le rôle critique de bâtisseur de ville. Le projet assiste des leaders à formuler l'impératif d'immigration de leur communauté et à promouvoir les bienfaits sociaux et économiques de l'immigration.

On a besoin du leadership de la ville pour aider la communauté élargie à accepter que la qualité de vie que cherchent les immigrants et dont tous les résident ont envie serait à trouver dans une base économique, sociale et culturelle vraiment dynamique. Quelle que soit leur taille ou leur histoire, les villes réussies ont ceci de commun : des gouvernements innovateurs et prévoyants qui savent utiliser l'autorité et les instruments de la charge publique pour mieux servir les intérêts de tous, y compris des nouveaux arrivants. Si l'on réussit à innover au niveau municipal, on peut dès lors réorienter le discours public en ce qui concerne les bienfaits de limmigration et favoriser le développement de meilleurs systèmes de mobilité.

\section{BONNE IDÉE Nº 1}

\section{La Stratégie d'immigration du Grand Moncton Moncton (NB)}

De nouveaux leaders font surface au niveau municipal pour aborder les questions d'attirance et de rétention. À Moncton, par exemple, on considère l'immigration comme un pilier de la stratégie de développement économique. Selon la Stratégie d'immigration du Grand Moncton (2014-2018) : " La croissance démographique n'est pas une fin en soi. La croissance économique non plus ... les centres urbains continuent à impulser la croissance économique et démographique non pas pour revendiquer des droits dans l'abstrait de s'en vanter mais parce qu'elle est nécessaire au soutien de la capacité fiscale du gouvernement de fournir des services publics et de linfrastructure. " (1)

\section{Votre ville a-t-elle une stratégie d'immigration?}




\section{BONNE IDÉE N 2}

\section{Pouvoir d'achat Toronto (ON)}

Les municipalités qui montre la voie se servent du pouvoir d'achat et de la diversité des fournisseurs pour diriger la croissance inclusive et la prospérité urbaine. Tous les ans, la ville de Toronto dépense environ 1,8 milliards de dollars canadiens (1,35 milliards de dollars US) en biens et services, depuis de grands projets de construction jusqu'à des contrats de restauration isolés. À compter de 2017, la ville veut exploiter ce pouvoir d'achat pour permettre à des minorités, des indigènes, des immigrants récents et des personnes handicapées de sortir de la pauvreté. La politique d'approvisionnement social de Toronto vise à aider à participer au processus d'appel d'offres les entreprises dont les propriétaires sont membres de groupes défavorisés. Les fournisseurs sous contrat avec la ville sont encouragés à prendre part aux programmes de développement de la main-d'œuvre auprès de groupes vulnérables tels que les jeunes.

\section{Votre ville aurait-elle envie d'adopter une telle politique d'approvisionnement social pour favoriser l'emploi de nouveaux arrivants?}

\section{Adopter une approche pancommunautaire de planification}

Le Cadre d'Avenirs Immigrants ne s'arrête pas à des politiques bien faites ou des analyses de données ; il propose des stratégies qui prennent la forme de relations sociales positives se manifestant dans nos institutions ordinaires. Un fait pertinent, c'est que dans les villes de migration récemment devenues des portes d'entrée il n'existe aucun soutien à l'installation. Les immigrants ont besoin d'une infrastructure de soutien. Et au Canada en principe cela veut dire des services d'installation. Par contre, dans des nouvelles portes d'entrée - ou faute d'un secteur d'installation bien établi - il y a d'autres actifs communautaires capables de combler la lacune, tels que les institutions essentielles, ancrées ou fondées sur le milieu. Il faut penser, par exemple, à des écoles et la prestation d'une formation linguistique ou à des bibliothèques et la centralisation des informations concernant le logement ou à des services communautaires et la prestation de conseils d'affaires dont auraient besoin les entreprises en démarrage. Voici des services qui, avec un peu de modification, pouvaient accorder un soutien global à tous les membres de la communauté.

Une approche pancommunautaire signifie qu'on se sert des actifs communautaires existants pour subvenir aux besoins d'une population de plus en plus diverse. Cela peut donner naissance à un nouveau localisme, un type de leadership civique apte à exploiter les talents des agents de changement régionaux ainsi qu'à favoriser un travail communautaire durable. Du fait de s'impliquer dans un processus multipartite de planification communautaire - de créer un écosystème de soutien - on se rend capable également de faciliter la constitution d'une infrastructure de soutien. C'est ainsi que des leaders municipaux peuvent évaluer - pour ensuite choisir - des solutions répondant aux besoins en mutation d'une communauté en pleine croissance. 
En plus, l'approche pancommunautaire met en lumière la capacité d'acteurs non traditionnels, tels que les employeurs, les entrepreneurs et les étudiants internationaux, de s'intéresser à des questions d'immigration aussi bien que l'importance qu'il y a à permettre à des immigrants de participer au processus de planification et aux activités communautaires. Le processus de planification s'avère plus robuste et plus résilient si la communauté d'accueil est invitée à collaborer sur les stratégies de l'intégration d'immigrants.

Une approche pancommunautaire doit être flexible afin que des leaders, en rédigeant leur plan, puissent s'y accommoder non seulement de la communauté et ses besoins immédiats et changeants mais aussi de l'économie et son évolution imprévisible. Il vous faut de la réflexion à long terme pour envisager des approches novatrices à la planification communautaire et la prospérité économique de votre région.

\section{BONNE IDÉE N 3}

\section{Nouvelles conversations Fredericton (N.B.)}

Le Nouveau-Brunswick a toujours eu un faible taux d'immigration. À l'échelle nationale, une personne sur cinq est né à l'étranger tandis qu'au Nouveau-Brunswick le chiffre serait plutôt une personne sur vingt. Et pourtant, selon les enquêtes. la plupart des Néo-Brunswickois se considèrent aussi diversifiés que le reste du pays. Cette idée fausse a révélé une lacune majeure dans la compréhension des liens existants entre la population, la croissance économique et l'u impératif immigrant ". La tournée " Nouvelles conversations " a été effectuée justement pour relever ces défis.

La tournée " Nouvelles conversations ", effectuée dans beaucoup de villes partout dans la province, est la première en son genre : un forum public sur la croissance économique, la subsistance communautaire et l'immigration. En 2018, grâce à une subvention d'IRCC, on a pu visiter 15 municipalités pour s'en entretenir avec un large éventail d'intervenants, y compris les employeurs, les leaders municipaux, les agences communautaires et le grand public. L'un des résultats majeurs de ces forums à été l'emphase bien plus fréquente dans ce que racontaient les médias locaux et provinciaux sur l'immigration comme une solution positive pour le marché du travail. Par surcroît, la ville de Moncton et deux autres régions sont en train d'élaborer des stratégies de croissance basées sur l'immigration.

\section{Votre ville a-t-elle une stratégie de sensibilisation du public pour s'entretenir de l'avenir démographique et économique?}


BONNE IDÉE N 4

\section{La Rural Employment Initiative Région de Peel (Ontario)}

Les communautés rurales de l'Ontario ont besoin de nouveaux arrivants - et on élabore de nouveaux programmes pour y apporter du soutien. Mais qu'en est-il des nouveaux arrivants qui se sont déjà installés au Canada ? La migration secondaire peut servir de stratégie dynamique pour attirer ceux-ci à des communautés rurales. Beaucoup de professionnels talentueux formés à l'étranger habitent la région du Grand Toronto. Accepteraient-ils de se déménager, eux et leurs familles, dans une communauté rurale, ils en seraient des atouts.

En reconnaissance de cette réalité, en 2016 le Newcomer Centre of Peel (NCP), en partenariat avec l'Association des Sociétés d'aide au développement des collectivités de l'Ontario, a instauré son Initiative concernant l'emploi rural (Rural Employment Initiative, ou REI).

Le projet REl collabore avec des nouveaux arrivants pour leur trouver de l'emploi et du logement dans les communautés rurales. À noter qu'il collabore aussi avec ces dernières pour créer des conditions d'accueil.

Avez-vous eu l'idée d'attirer des nouveaux arrivants à votre communauté grâce à la migration secondaire?

\section{Comment se décider sur ceux qui va s'impliquer dans le processus de planification ? Se concentrer sur les principaux acteurs économiques}

Le cadre que nous proposons se base sur l'idée que même si les résultats économiques et l'intégration au marché du travail sont d'une importance primordiale à l'égard de l'expérience d'immigration, cela va compter pour peu sans que la communauté offre du soutien et favorise le sentiment d'appartenance. À cette fin, le cadre vise à encourager la participation civile de tous les résidents.

Dans notre approche nous sollicitons l'aide d'intervenants tels que les employeurs et les entrepreneurs et, à une plus grande échelle, d'institutions publiques telles que les bibliothèques, les hôpitaux et les écoles pour cultiver le sentiment d'appartenance par l'intermédiaire de politiques progressifs, d'initiatives et de stratégies. C'est notre objectif de créer une infrastructure de soutien qui en même temps qu'elle s'occupe des besoins de nouveaux arrivants, avant et après leur arrivée, contribue à un processus d'urbanisme se fondant sur des principes d'inclusion conçus pour qu'aucun membre de la communauté ne subisse de l'exclusion, de la discrimination ou du racisme. 


\section{Pourquoi les emplois ne suffisent-ils plus?}

\section{Ce sont les immigrants qui font le choix.}

Plus de gens se déplacent aujourd'hui que jamais. Auparavant, la migration a été gouvernée par les pays destinataires qui, en filtrant les candidats, n'en ont choisi que les plus éminents. Maintenant, en revanche, ce sont les immigrants qui font le choix. Le plus souvent ils prennent en charge le processus de sélection eux-mêmes, en se servant d'un éventail élargi de pays destinataires et en votant, comme on dit, avec les pieds. En d'autres mots, le marché acheteur s'est transformé en un marché vendeur sur lequel les migrants sont courtisés à cause de leur argent, leurs compétences et leur talent. Il est bien évident qu'on doit rendre plus rapide et plus efficace la méthode dont leur sont fournis lintégration et linclusion. Voire, les meilleures stratégies d'intégration visent à améliorer l'attractivité de la ville auprès de migrants potentiels et à réunir les conditions propices à leur investissement et leur appartenance.

\section{LE RÔLE DES EMPLOYEURS}

Nous croyons que le secteur d'affaires va s'imposer de plus en plus fréquemment comme partenaire dans la planification communautaire ainsi que l'attirance et la rétention d'immigrants. Personne ne sait mieux que les employeurs les défis associés à l'attirance et l'incubation de talent. Ils comprennent bien l'argument commercial pour la diversité - comment elle peut impulser l'innovation sur un marché mondial hautement concurrentiel. Dans ce modèle, c'est à eux de formuler l'immigration comme une stratégie essentielle au succès du développement de l'économie locale. Et cela signifie tous les employeurs, n'importe la taille de leur entreprise ni celle du bassin de talents.

Certes, les employeurs, qu'ils soient du secteur public ou privé, ont un rôle à jouer par rapport à l'attirance et la rétention d'immigrants. Bien qu'ils prennent part à divers programmes d'immigration fédéraux et provinciaux visant à remédier aux pénuries de main-d'œuvre, ils restent néanmoins conscients que la possibilité d'un bon emploi ne suffit plus à elle seule pour attirer des nouveaux arrivants. Ils ont donc envie que leurs efforts soient dirigés vers un éco-système de soutien plus élargie comprenant d'autres intervenants et des partenariats appropriés. Face à un marché du travail en évolution accélérée, les employeurs se donnent le droit maintenant d'agir de façon délibérée et stratégique sur le domaine d'immigration et d'installation. C'est surtout le cas dans les villes petites et moyennes où l'on relève ces défis d'une manière unique.

"Il y a des employeurs pionniers dans la région dont les succès en matière d'intégration peuvent être imités - de tels efforts doivent être salués par des leaders communautaires pour que d'autres employeurs puissent en apprécier la valeur même s'ils ne sont pas nécessairement les premiers à adopter les programmes de diversité. "

Rural Ontario Institute 2018 


\section{L'ENTREPRENEURIAT MIGRANT}

Aux yeux des immigrants, l'entrepreneuriat a toujours été un moyen de subvenir à leurs besoins. Toutefois, les difficultés relatives au démarrage d'une entreprise, y compris le manque d'accès au financement ou à l'espace commercial nécessaire, se sont avérées insurmontables pour beaucoup d'entre eux. Bien sûr, on rencontre les mêmes difficultés partout. Quel que soit le service, le produit ou le marché, la liste de contrôle en toute probabilité va inclure des questions techniques épineuses touchant les permis et le zonage, les lois locales du travail, la conformité fiscale, la vérification et les systèmes financiers, pour n'en citer que les plus évidentes. D'autres difficultés peuvent découler du fait d'avoir une connaissance limitée des marchés locaux ou de ne pas avoir un accès suffisant aux réseaux d'affaires essentiels. Il y a d'excellents services commerciaux prêts à aider les nouvelles entreprises en démarrage, mais des différences culturelles ou des barrières linguistiques peuvent empêcher des entrepreneurs en devenir de prendre le premier pas si important, soit de s'y adresser.

Les villes petites et rurales se rend de plus en plus compte des avantages économiques, démographiques et sociaux que leur apporte l'immigration. Pour en attirer il faut un environnement accueillant et solidaire. Les nouveaux arrivants reconnaissent que les grandes agglomérations ne sont pas les seuls endroits où trouver du succès.

\section{BONNE IDÉE NO 6}

\section{Investir dans le succès Fredericton (Nouveau-Brunswick)}

La ville de Fredericton (Nouveau-Brunswick) a embrassé l'immigration comme une stratégie critique visant à contrer son déclin démographique. Pour en faire croître et la population et l'économie, on a misé sur l'entrepreneuriat. Comme le montre le Programme de mentorat pour les entrepreneurs qu'on a inauguré en 2009. Grâce à celui-ci, des nouveaux arrivants reçoivent de l'aide pour l'enregistrement des entreprises, la comptabilité, les impôts, la connaissance de l'économie locale et davantage encore. Le succès du programme se mesure à l'aune de nombreux facteurs, y compris la création des emplois, la rétention de nouveaux arrivants, l'évolution du réseau entrepreneurial des immigrants et enfin la reproductibilité de ses résultats. Beaucoup de programmes se sont inspirés du Programme de mentorat. Par exemple, l'accélérateur d'entreprises La Ruche, une installation de cohabitation lancée en 2014 et située dans un district de biens immobiliers de premier ordre. Là les entrepreneurs immigrants ont accès jour et nuit à de l'espace et se trouvent fournis, entre autres choses, d'une adresse commerciale, d'une boîte aux lettres et d'une affiliation à la chambre de commerce.

Votre ville a-t-elle des programmes de mentorat ou de l'accélération d'entreprises qui peuvent soutenir des nouveaux arrivants aussi bien que des résidents? 


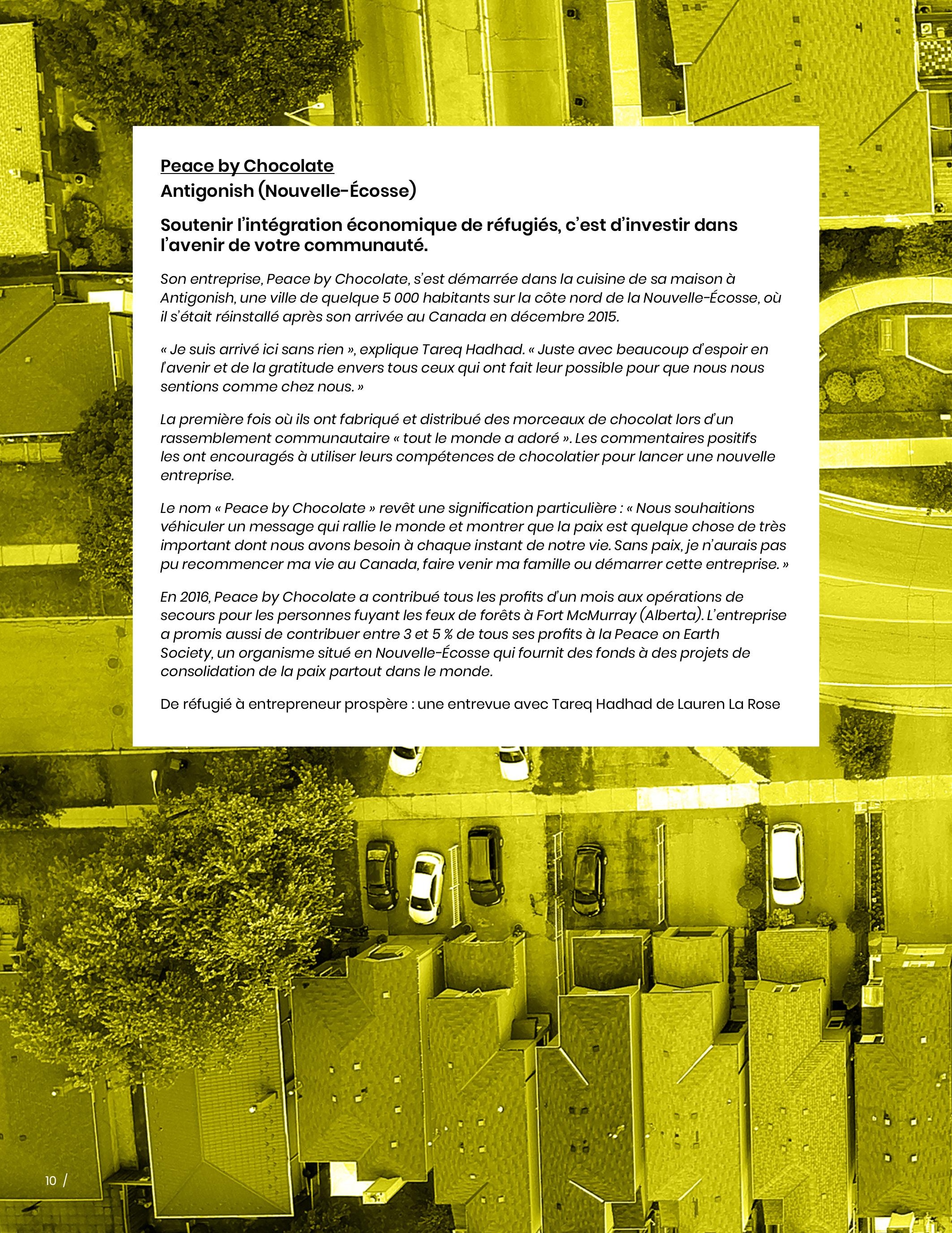




\section{LE RÔLE DES COLLÈGES ET DES UNIVERSITÉS}

Un grand nombre d'économistes considèrent les étudiants internationaux comme des sources majeures de capital social et économique. Dans ce contexte, les universités jouent un rôle unique en matière d'immigration, parce que les étudiants internationaux qu'ils attirent et choisirent sont aptes à devenir ensuite des immigrants et de futurs citoyens idéals.

Selon la "Stratégie en matière d'éducation internationale " (2014, p.11) du gouvernement du Canada : " Les étudiants internationaux sont des candidats idéals pour immigrer au Canada étant donné qu'ils détiennent des diplômes canadiens, qu'ils maîtrisent au moins l'une des langues officielles et qu'ils ont, pour la plupart, une expérience de travail canadienne pertinente. " Déposée par le ministère des Affaires étrangères, du Commerce et du Développement, la stratégie s'est engagée à atteindre un objectif ambitieux, celui de doubler le nombre d'étudiants internationaux avant l'an 2022. L'un des buts principaux en était d'assurer que ladite augmentation contribue à " la croissance économique et la prospérité de chaque région du pays " (Le gouvernement du Canada, 2014, p. 11). Toutefois, il y a eu, d'après des recherches récentes, une dispersion inégale des étudiants internationaux entre les provinces et les territoriaux du pays. Ce qui est problématique, parce qu'elle ajoute à la distribution inégale du capital social et économique.

On attribue aux étudiants internationaux un rôle de plus en plus signifiant à l'égard de la prospérité urbaine. Ils peuvent être des immigrants et des employés dans une société vieillissante ; ils peuvent offrir un accès futur à des marchés globaux ainsi que des compétences interculturelles; et ils peuvent aider à ouvrir une communauté et rendre sa population plus accueillante et plus diverse.

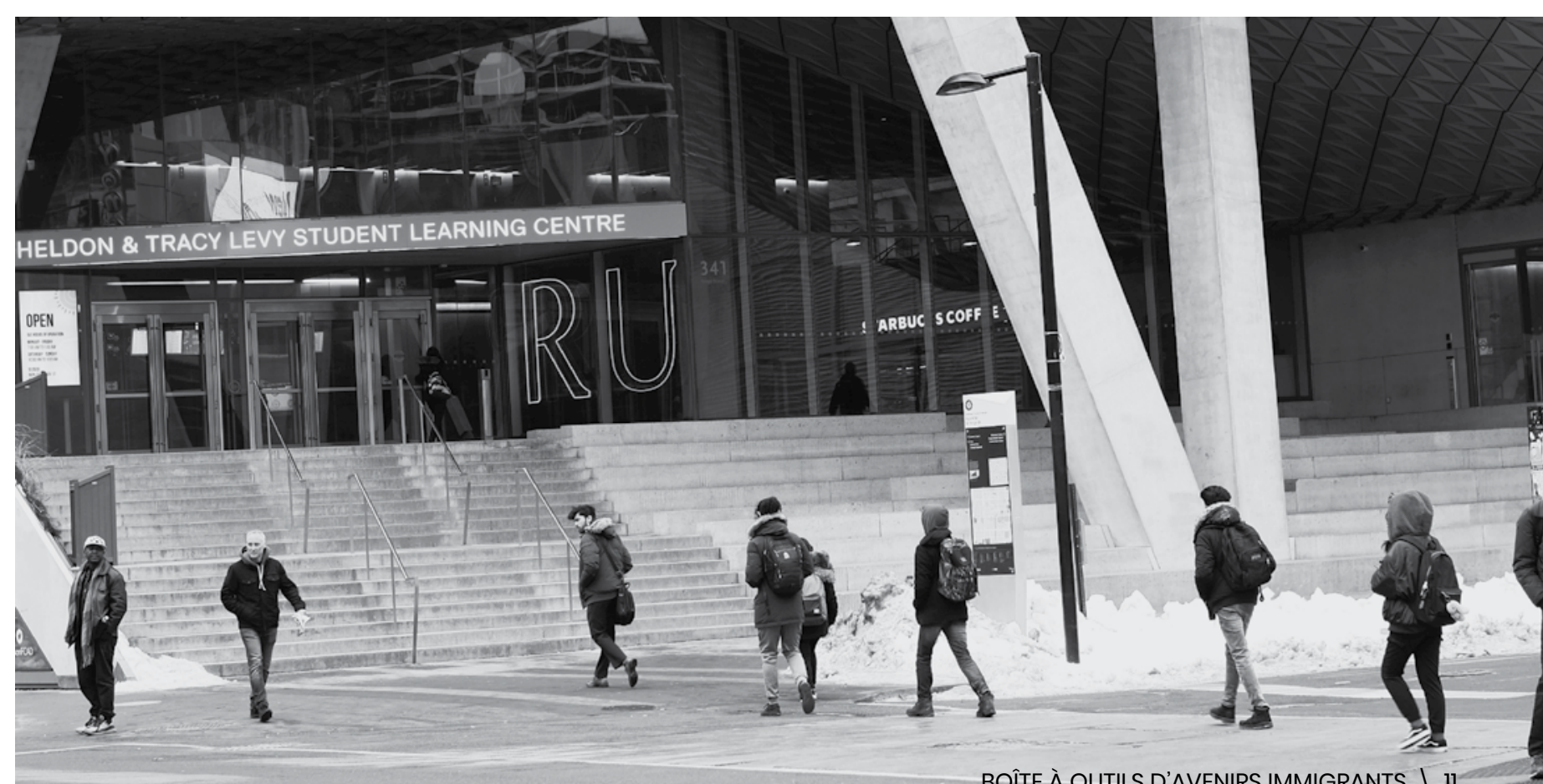



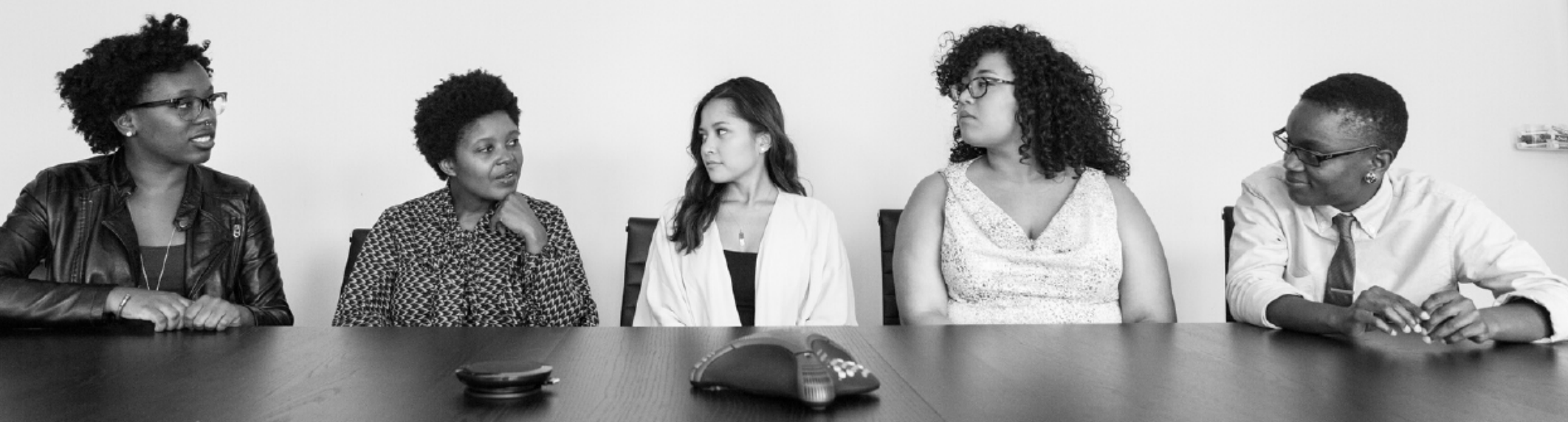

BONNE IDÉE No 7

\section{L'apprentissage par la pratique, Ryerson University Lifeline Syria Challenge Toronto (Ontario)}

Un programme de parrainage à l'intention des réfugiés mené par l'université fournit des possibilités d'apprentissage par la pratique à des étudiants, des bénévoles et des nouveaux partenariats institutionnels dans la ville

Le Ryerson University Lifeline Syria Challenge (RULSC) a été instauré en juillet 2015 dans le but d'aider les étudiants à devenir des forces motrices de la réinstallation de réfugiés à titre privé. Le premier organisme de ce type au Canada, le RULSC a dû se mettre au travail rapidement pour consolider dans le cadre existant de parrainage privé une infrastructure où se tiennent compte les besoins d'étudiants, de parrains privés et de réfugiés syriens récemment arrivés.

À l'origine, l'idée était de s'appuyer sur l'expertise, les compétences et l'énergie des étudiants pour créer un modèle d'équipe relatif au parrainage de réfugiés que l'on pourrait alors mettre à l'échelle d'autres universités et institutions au Canada. D'anciens étudiants de Ryerson et des membres de la communauté se sont réunis en équipes d'au moins cinq personnes pour assumer les responsabilités - qu'elles soient financières ou liées à l'installation - du parrainage de réfugiés à titre privé. II incomberait aux étudiants d'apporter du soutien aux équipes et aux familles de nouveaux arrivants sans en être eux-mêmes formellement responsables.

En exploitant les compétences et l'entrain des étudiants et leur nouvelle perspective sur ce qu'il faut pour faire de Toronto une communauté accueillante, le RULSC a réussi à se déployer sur trois autres campus universitaires : l'Université de Toronto, l'Université de York et I'OCAD U.

\section{Que fait le collège ou l'université de votre région en matière d'immigration ?}




\section{BONNE IDÉE N 8}

\section{Maximiser les chances de succès de jeunes réfugiés, SchlaU-Schule Munich (Allemagne)}

La SchlaU-Schule n'a rien d'ordinaire. Cette "école intelligente " a été fondée en 2000 par Michael Stenger pour combler une lacune du système d'asile de l'Allemagne à cause de laquelle les jeunes réfugiés âgés de plus de 16 ans ont été empêchés d'aller à l'école, ce qui a eu pour effet de leur refuser le droit à l'éducation et de les exclure d'une première étape importante du processus d'installation.

Le curriculum de la SchlaU offre une approche globale à de jeunes réfugiés et des chercheurs d'asile non accompagnés âgés entre 16 et 25 ans. Les cours sont équivalents à ceux offerts par les écoles publiques, et les programmes répondent aux besoins les plus pressants des jeunes. En leur fournissant de la formation linguistique spécialisée, des programmes d'enseignement courants, de l'assistance juridique et sociale et du soutien psychologique et pédagogique, on les prépare à réussir aux examens de base des écoles publiques, ce qui les permet à se qualifier pour des études post-secondaires ou de l'apprentissage professionnel et éventuellement une carrière professionnelle.

\section{Quels sont les soutiens offerts par le collège ou l'université de votre région à des étudiants internationaux?}

\section{BONNE IDÉE Nº 9}

\section{Les étrangers deviennent des amis Erfurt (Allemagne)}

Ville moyenne au sud-ouest de Leipzig dans l'ancienne Allemagne de l'Est, Erfurt a rétabli son université il y a trente ans, après la réunification. Bientôt elle a commencé à attirer un grand nombre d'étudiants du monde entier pour qui c'était une destination relativement abordable et hautement souhaitable. Les leaders municipaux se sont vite rendus compte qu'il leur fallait embrasser cette population d'étudiants internationaux et la traiter comme un moteur de croissance. Le conseil municipal et l'Université d'Erfurt, ainsi que l'école polytechnique et le collège communautaire, ont entrepris un projet pour faire connaître leur esprit d'ouverture et leur culture d'accueil, dans l'espoir d'établir la réputation de la ville en tant que capitale régionale tolérante et sympathique.

Le résultat en a été Fremde werden Freunde (Les étrangers deviennent des amis). Le programme met des étudiants internationaux en contact avec des résidents locaux pour les aider à se sentir chez eux et pour élargir les horizons culturels de tous. Les leaders d'affaires ne tardaient pas à accepter l'intégration des étudiants dans l'économie locale. Voire, en 2006, le programme a été augmenté pour inclure toute entreprise de la ville qui serait prête à agir comme mentor et à offrir des stages. Grâce à la participation de la chambre de commerce, on a pu y ajouter des visites sur place, des foires aux emplois et des événements professionnels. Maintenant les entreprises peuvent puiser dans un bassin de jeunes gens talentueux qui sont dotés de compétences techniques, linguistiques et interculturelles ainsi que d'un accès potentiel aux marchés globaux.

\section{Tout le monde profite de l'inclusion sociale}




\section{INSTITUTIONS PRINCIPALES}

Pour créer les conditions d'inclusion, une infrastructure de soutien dépend de la participation et la transformation d'institutions publiques ou d'industries locales existantes, telles que les hôpitaux, les écoles et les bibliothèques. Cela peut prendre la forme de politiques des $\mathrm{RH}$ inclusives, de ressources en plusieurs langues ou dinitiatives telles que la formation linguistique sur le terrain. Dans notre approche pancommunautaire, nous mettons en valeur la contribution d'un nombre varié d'intervenants à la création de conditions informelles d'investissement.

\section{BONNE IDÉE No 10}

\section{"Welcomed in Halifax" Halifax (N.É.)}

Un laissez-passer de transport en commun offert gratuitement aux nouveaux arrivants pour leur permettre d'accéder à des services municipaux, de se rendre à l'école ou au travail et d'explorer la ville en même temps que ceux qui sont devenus leurs voisins.

Le Bureau municipal de la diversité et de l'inclusion de Halifax, qui héberge aussi le Partenariat local d'immigration, a fait quelques recommandations sur la meilleure façon de soutenir des réfugiés. Le conseil régional reconnaissait que " l'attraction et la rétention des réfugiés peuvent aussi contribuer à la vigueur de l'économie et la communauté et s'aligner sur les directives précédentes du conseil. "

Peut-être l'initiative la plus concrète, c'était le laissez-passer " Welcomed in Halifax ". Valable un an, "Welcomed in Halifax " donne à des réfugiés nouvellement arrivés libre accès aux transports en commun et aux programmes et installations de loisirs municipaux (y compris des leçons de natation, des camps de vacances et beaucoup d'autres activités convenables pour tous les membres de la famille). On utilise le laissezpasser également pour obtenir l'entrée gratuite à des institutions culturelles locales comme, par exemple, les musées et le Centre des Jeux du Canada ainsi que l'accès gratuit à leurs programmes.

Établi à temps pour l'arrivée des réfugiés syriens en décembre 2015, début 2016 le programme se déroulait sans à coups. Selon Roberto Montiel, coordinateur du Partenariat local d'immigration, l'intention a été de mettre le laissez-passer à la disposition de tous les réfugiés et non seulement les Syriens nouvellement arrivés. Depuis 2017, y sont admissibles également les revendicateurs du statut de réfugié. La ville a pu distribuer les cartes WIH par l'entremise de l'Immigration Services Association of Nova Scotia (ISANS) et de la Halifax Refugee Clinic.

Dans quelle mesure les services publics de votre ville sont-ils accessibles? 


\section{Naviguer un système de santé pour nouveaux arrivants, Le Centre de santé pour nouveaux arrivants d'Ottawa Ottawa (Ontario)}

L'installation et la santé des immigrants vont de pair. Une réalité que reflète le Centre de santé pour nouveaux arrivants d'Ottawa.

C'est un partenariat entre le CCI Ottawa, un organisme multiservices local desservant les immigrants et les réfugiés, et le Centre de santé communautaire Somerset Ouest. Là on fournit des services culturellement compétents d'intégration et de santé et, du fait que les trois centres se situent dans le même bâtiment, on est capable d'opérer des transitions sans heurt entre des systèmes de service très différents,

Dans une clinique médicale complète les nouveaux arrivants peuvent recevoir des soins de santé à court terme pendant qu'ils cherchent un médecin ou une équipe soignante qui les prendra en charge de façon permanente.

Le programme Intervenant-Pivot Santé se compose d'un personnel multilingue spécialement formé pour aider des nouveaux arrivants à naviguer le système de santé local. Le Centre s'est modelé sur la Multicultural Health Brokers Co-op d'Edmonton (Alberta). Ici à Ottawa on admet tout client ayant besoin d'aide pour naviguer à travers le système de santé. Cette admissibilité a été importante. On trouve que même les clients de retour après une longue absence ont quelquefois besoin, eux aussi, d'y être aidés.

L'un des buts principaux du personnel du Centre est de renseigner des nouveaux arrivants sur le système de santé de façon à leur permettre de le naviguer en toute indépendance. On ne veut pas des clients à long terme à la clinique. On est là plutôt pour les aider à trouver des prestataires de services de santés permanents. Les clients ont accès au programme pour une durée maximale de deux ans. Et ça marche : en moyenne, ils en font usage durant 14 mois. À la fin, ils ont suffisamment compris le système pour agir de leur propre initiative en accédant aux services.

\section{Que fait l'établissement de soins de santé local pour soutenir des nouveaux arrivants?}
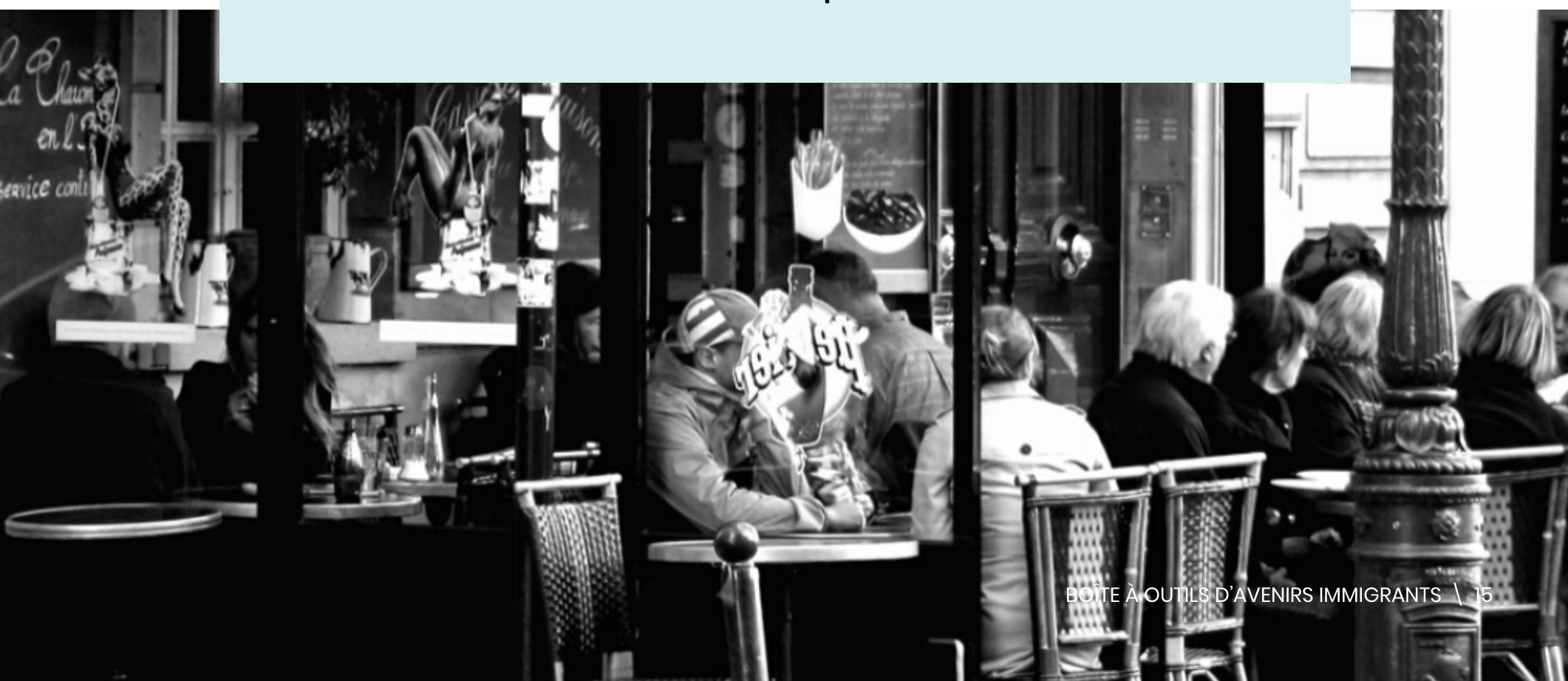


\section{Conclusion}

Les villes comptent pour beaucoup. Les gouvernements municipaux sont responsables d'un grand nombre de choses qui ont un effet sur notre vie quotidienne - les rues, les bibliothèques, les conseils scolaires, les policiers, les piscines, les terrains de foot .... Ils montrent la voie en tant que décideurs politiques, prestataires de services, employeurs, vendeurs et acheteurs de services, producteurs de richesses et moteurs de l'économie.

Les immigrants font le choix. Plus de gens se déplacent aujourd'hui que jamais. Auparavant, la migration a été gouvernée par les pays destinataires qui, en filtrant les candidats, n'en ont choisi que les plus éminents. Maintenant ce sont les immigrants qui font le choix.

Les stratégies d'intégration les plus efficaces se concentrent sur l'attractivité des villes pour des migrants potentiels et la création des conditions d'investissement et d'appartenance.

La diversité, c'est un fait. L'inclusion, c'est un choix. Une ville compétente en matière d'immigration n'attend pas d'être contactée par les migrants ; elle est la première à agir, en assurant qu'on parle la même langue et qu'on peut les satisfaire quand ils cherchent des services ou veulent participer à des processus publics.

Et la ville est, bien sûr, une entité représentative. Elle nous comprend tous, nous le peuple. Donc, un glissement de l'intégration vers l'inclusion implique la participation et l'engagement de tout le monde. L'engagement d'un nombre varié d'intervenants dans toute une série de secteurs peut créer une ville non seulement accueillante aux immigrants mais compétente en matière d'immigration. 


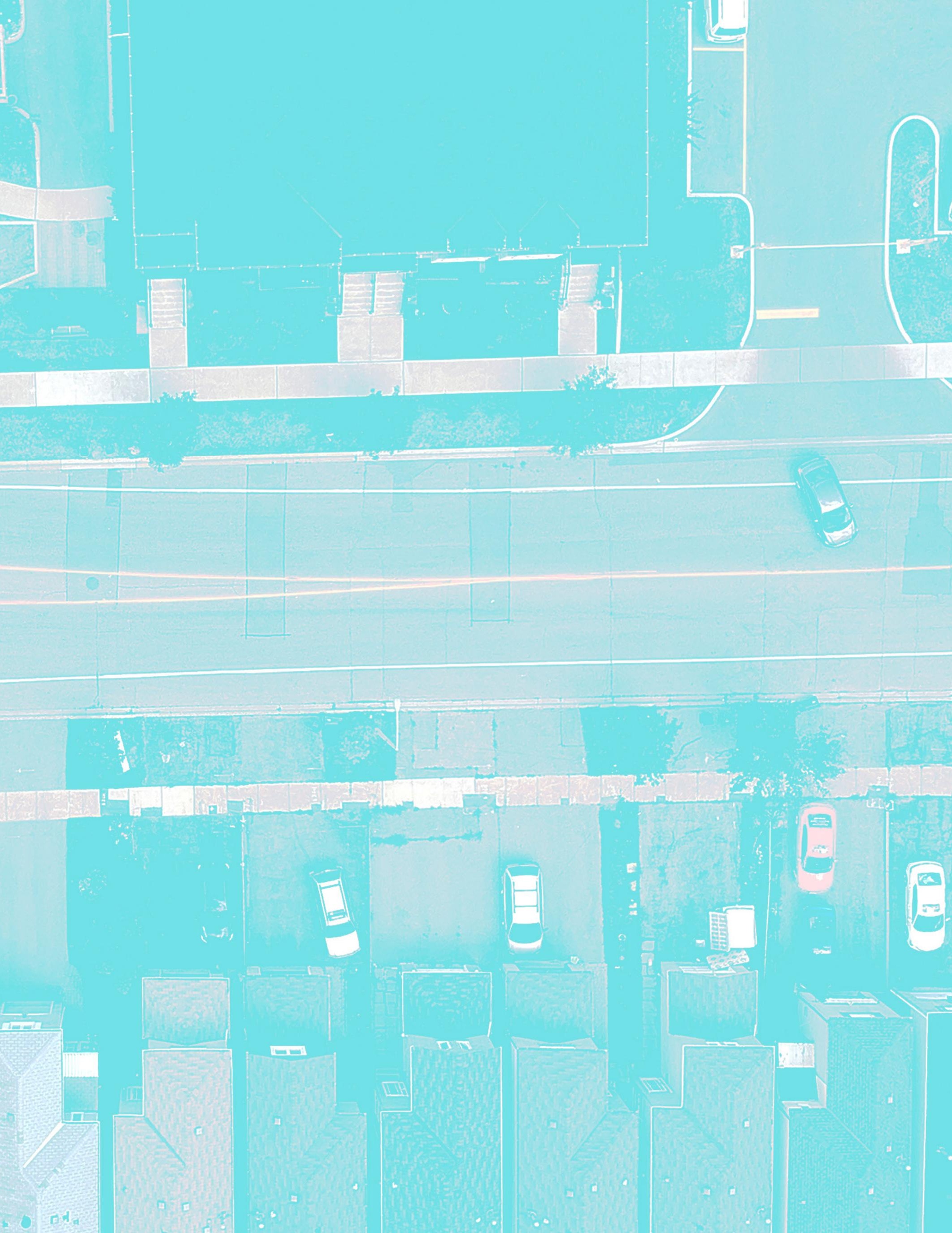

